\title{
LGR6 promotes glioblastoma malignancy and chemoresistance by activating the Akt signaling pathway
}

\author{
YUAN YUAN CHENG ${ }^{1}$, XUE YANG $^{1}$, XIN GAO $^{2}$, SI XIN SONG ${ }^{3}$, MING FENG YANG $^{4}$ and FANG MIN XIE ${ }^{3}$ \\ ${ }^{1}$ Department of Oncology, Tai'an Central Hospital, Tai'an, Shandong 271000; ${ }^{2}$ Department of Neurosurgery, \\ The Affiliated Hospital of Qingdao University, Qingdao, Shandong 266100; ${ }^{3}$ Department of Neurosurgery, \\ The Second Affiliated Hospital of Shandong First Medical University and Shandong Academy of Medical Sciences; \\ ${ }^{4}$ Institute of Basic Medicine of Shangdong, First Medical University and Shandong Academy of Medical Sciences, \\ Tai'an, Shandong 270000, P.R. China
}

Received November 8, 2019; Accepted June 17, 2020

DOI: $10.3892 / \mathrm{etm} .2021 .10798$

\begin{abstract}
Chemoresistance is the primary cause of the poor outcome of glioblastoma multiforme (GBM) therapy. Leucine-rich repeat-containing G-protein coupled receptor 6 (LGR6) is involved in the growth and proliferation of several types of cancer, including gastric cancer and ovarian cancer. Therefore, the aim of the present study was to investigate the role of LGR6 in GBM malignancy and chemoresistance. Cell counting kit- 8 and Matrigel ${ }^{\circledR}$-Transwell assays were conducted to assess GBM cell viability and invasion. The effect of LGR6 on cell cycle progression and activation of Akt signaling was analyzed by performing propidium iodide staining and western blotting, respectively. The results demonstrated that LGR6, a microRNA-1236-3p target candidate, promoted GBM cell viability and invasion, and mediated temozolomide sensitivity in SHG-44 and U251 GBM cells. In addition, LGR6 triggered the activation of the Akt signaling pathway during GBM progression. Collectively, the results of the present study suggested that LGR6 promoted GBM malignancy and chemoresistance, at least in part, by activating the Akt signaling pathway. The results may aid with the identification of a novel therapeutic target and strategy for GBM.
\end{abstract}

\section{Introduction}

Glioblastoma multiforme (GBM) is the most lethal primary brain tumor (1) worldwide, with a mean survival time of 8-12 months (2). The current clinical strategy for GBM consists of surgical resection, radiation therapy and treatment with adjuvant temozolomide (TMZ) chemotherapy (3).

Correspondence to: Dr Fang Min Xie, Department of Neurosurgery, The Second Affiliated Hospital of Shandong First Medical University and Shandong Academy of Medical Sciences, 289 Linshan Street, Tai'an, Shandong 270000, P.R. China

E-mail: xiefm_smu@163.com

Key words: chemotherapy, glioblastoma, MK-2206, temozolomide
Although TMZ exhibits antitumor effects against high-grade glioma (4), previous studies have suggested that its efficacy is affected by the development of drug resistance in tumor cells (5-7). Therefore, identifying the mechanism underlying TMZ resistance and developing a new adjuvant chemotherapy drug against GBM is important.

Leucine-rich repeat-containing G-protein coupled receptor 6 (LGR6) is involved in the growth and proliferation of multiple types of cancer, including colon cancer and gastric cancer (8-10), and high levels of LGR6 have been correlated with colorectal metastasis (8). LGR6 was initially identified as a cognate receptor of R-spondin ligands, which serve as enhancers of WNT signaling (11-13) and was later identified as a stem cell marker (14-17). Functioning as an oncogene or tumor suppressor, LGR6 modulates the activation of signaling pathways, such as the zinc transporter ZIP10-p63 (18) and WNT (19) signaling pathways.

In addition, several signaling pathways, including the STAT5 and PI3K/Akt signaling pathways, serve a vital role during the progression of GBM (20-22). Cytokine-induced Janus kinases initiate the STAT family or activate mitogen-activated protein kinases PI3K and mTOR (23), which are all associated with the progression of GBM (24-27); therefore, assessing whether LGR6 can activate these signaling pathways and serve as a potent therapeutic target for GBM requires investigation.

\section{Materials and methods}

Cell culture. Human GBM cell lines T98G (accession no. CVCL_0556) and U87 (glioblastoma of unknown origin; accession no. CVCL_0022) were purchased from American Type Culture Collection. GBM cell lines SHG-44, U251 and human normal glial HEB cells and human embryonic kidney 293T cells were purchased from The Cell Bank of Type Culture Collection of the Chinese Academy of Sciences. T98G and U87 cells were maintained in modified Eagle's medium (MEM; Hyclone; Cytiva), SHG-44 cells were maintained in RPMI-1640 medium (Hyclone; Cytiva) and U251, HEB and 293 T cells were maintained in DMEM (Hyclone; Cytiva). All culture mediums were supplemented with $10 \%$ FBS (Hyclone; Cytiva). Cells were maintained at $37^{\circ} \mathrm{C}$ in $5 \% \mathrm{CO}_{2}$ 
incubators. To establish TMZ-resistant cell lines, SHG-44 and U251 cells were cultured and passaged over 8 weeks in the presence of increasing concentrations of TMZ (30 to $300 \mu \mathrm{M}$; Selleck Chemicals) to generate $\mathrm{TMZ}$ resistant lines at $37^{\circ} \mathrm{C}$ in $5 \% \mathrm{CO}_{2}$ incubator, which were denoted as SHG-44TMZ+ and U251TMZ+ as per a previous study (28) and the parental cells were denoted ad SHG-44TMZ- and U251TMZ-.

Plasmid construction and cell transfection. overexpression plasmids (LGR6) were constructed by inserting the LGR6 coding sequence into a pcDNA3.1 plasmid (General Biosystems, Inc.). An empty pcDNA3.1 vector was used as the negative control (Vector). The small interfering (si)RNA targeting LGR6 (siRNA-LGR6) and the control (siRNA-Ctrl) were purchased from Shanghai GenePharma Co., Ltd. The microRNA (miR)-1236-3p mimic (miR-1236-3p) and scrambled oligonucleotides (miR-Ctrl) were purchased from Guangzhou RiboBio Co., Ltd. Sequences are presented in Table I. The day prior to transfection, $\sim 2 \times 10^{5}$ cells were plated in growth medium without antibiotics at a density of $30-50 \%$. Both siRNAs and miRNAs were transfected into cells at a final concentration of 100 nM using Lipofectamine ${ }^{\circledR} 2000$ (Thermo Fisher Scientific, Inc.), according to the manufacturer's protocol. DNA fragments containing the wild-type (WT) or mutated (Mut) miR-1236-3p 3'-untranslated region (3'-UTR) and their complementary fragments were cloned from SHG-44 cDNA. The annealed double-stranded DNA was then cloned into the dual-luciferase reporter gene vector psicheck-2 (Promega Corporation). The recombinant WT and Mut reporter gene vectors were named LGR6-3' UTR-WT and LGR6-3' UTR-Mut, respectively.

Potential microRNAs prediction. To investigate whether microRNAs regulated the expression of LGR6, the available complementary-based algorisms were predicted using TargetScan (www.targetscan.org/vert_72) and miRTarBase (mirtarbase.mbc.nctu.edu.tw/php/index.php). miR-1236-3p displayed a low mirSVR score $(-2.69)$ and was selected as a prediction microRNA.

Luciferase activity analysis. $293 \mathrm{~T}$ cells $\left(\sim 5 \times 10^{3}\right.$ cells/well $)$ were plated in 96-well plates and co-transfected with $25 \mathrm{ng}$ luciferase reporter gene vector and $50 \mathrm{nM}$ miR-1236-3p or miR-Ctrl using Lipofectamine ${ }^{\circledR} 2000$ reagent (Invitrogen; Thermo Fisher Scientific, Inc.) according to the manufacturer's instructions. Following culturing at $37^{\circ} \mathrm{C}$ for $48 \mathrm{~h}$ in a $5 \% \mathrm{CO}_{2}$ incubator, luciferase activity were detected using the Dual-Luciferase Reporter assay system (Promega Corporation). The results were normalized to Renilla luciferase and analyzed, according to the manufacturer's protocol.

Cell viability. Cells were plated in 96 -well plates $\left(\sim 5 \times 10^{3}\right)$ and transfected with siRNA-LGR6, siRNA-Ctrl, LGR6 overexpression plasmids or empty pcDNA3.1 vector for $24 \mathrm{~h}$ at $37^{\circ} \mathrm{C}$ in $5 \% \mathrm{CO}_{2}$ incubator, then $\mathrm{TMZ}$ was added to culture medium at final concentrations of $0,100,200,300,400$ or $500 \mu \mathrm{M}$. At 0, 24, 48 and $72 \mathrm{~h}$ post-transfection, Cell Counting Kit-8 reagent (10 $\mu \mathrm{l}$; Beyotime Institute of Biotechnology) was added to each well for $4 \mathrm{~h}$ at $37^{\circ} \mathrm{C}$. The absorbance of each well was measured at a wavelength of $450 \mathrm{~nm}$ using the Multiskan GO plate reader (Thermo Fisher Scientific, Inc.).
Inhibitor treatment. Selective inhibitors of $\mathrm{Akt} 1 / 2 / 3$ (MK-2206) were purchased from Selleck Chemicals. Frozen aliquots $\left(-80^{\circ} \mathrm{C}\right)$ were melted and dissolved in DMSO (Sigma-Aldrich; Merck KgaA) and diluted in growth medium (RPMI-1640 medium for SHG-44 cells; DMEM medium for $\mathrm{U} 251$ cells). A total of $5 \mu \mathrm{M}$ MK-2206 was added to SHG-44 and U251 cells for $0,24,48$ or $72 \mathrm{~h}$ following transfection with LGR6 overexpression plasmids. Cell Counting Kit- 8 reagent (10 $\mu \mathrm{l}$; Beyotime Institute of Biotechnology) was added to each well for $4 \mathrm{~h}$ at $37^{\circ} \mathrm{C}$. The absorbance of each well was measured at a wavelength of $450 \mathrm{~nm}$ using a Multiskan GO plate reader (Thermo Fisher Scientific, Inc.).

Cell invasion. For cell invasion assays, Corning ${ }^{\circledR}$ Transwell ${ }^{\circledR}$ polycarbonate membrane cell culture inserts containing polycarbonate membranes with $8-\mu \mathrm{m}$ pores (Corning, Inc) were precoated with Matrigel ${ }^{\circledR}$ (BD Biosciences) for $30 \mathrm{~min}$ at $37^{\circ} \mathrm{C}$. Cells $\left(\sim 5 \times 10^{4}\right.$ cells/well $)$ were suspended in culture medium supplemented with 5\% FBS and plated into the upper chambers. The lower chambers were filled with culture medium supplemented with $20 \%$ FBS. Following incubation for $24 \mathrm{~h}$ at $37^{\circ} \mathrm{C}$ in $5 \% \mathrm{CO}_{2}$ incubators, cells were washed with PBS and fixed with cold $99.9 \%$ methanol for $30 \mathrm{~min}$ at room temperature. After staining with $1 \%$ crystal violet for $30 \mathrm{~min}$ at room temperature, cells on the upper surface of the membrane were removed using cotton swabs. Stained cells were counted using a light microscope and analyzed using Image $\mathbf{J}$ software (v18.0; National Institutes of Health).

Cell cycle assay. At $48 \mathrm{~h}$ post-transfection, cells were washed twice with cold PBS and harvested using trypsin. Cells were fixed with cold $75 \%(\mathrm{v} / \mathrm{v})$ ethanol overnight at $-20^{\circ} \mathrm{C}$. After washing twice with PBS, cells were suspended in staining buffer containing $5 \mu \mathrm{l}$ PI and $5 \mu \mathrm{l}$ RNAase A inhibitor for $30 \mathrm{~min}$ in the dark at room temperature using a Cell Cycle Analysis kit (Shanghai Yeasen Biotechnology Co., Ltd.), according to the manufacture's protocol. Stained cells were analyzed via ACEA NovoCyte flow cytometry instrument (ACEA Bioscience, Inc.) and cell cycle distribution was assessed using Novo Express software (https://www.aceabio. com.cn/support/software_download\#edit-group-novocytesoftware-download; ACEA Bioscience, Inc.).

Western blot analysis. Transfected cells were washed with cold PBS and total protein was extracted using RIPA lysis buffer (Beyotime Institute of Biotechnology) supplemented with phosphatase inhibitors (Roche Applied Science). Total protein was quantified using a bicinchoninic acid assay kit (Thermo Fisher Scientific, Inc.). Protein (30 $\mu \mathrm{g}$ per lane) was separated via 10\% SDS-PAGE and transferred onto PVDF membranes (Bio-Rad Laboratories, Inc.), which were blocked with $5 \%$ non-skimmed milk for $1 \mathrm{~h}$ at room temperature. The membranes were incubated overnight at $4^{\circ} \mathrm{C}$ with primary antibodies targeted against: Phosphorylated (p)-Akt (Ser473; dilution, 1:1,000; cat. no. 4060; Cell Signaling Technology, Inc.), Akt (dilution, 1:500; cat. no. OM238722; OmnimAbs), LGR6 (dilution, 1:1,000; cat. no. ab126747; Abcam) and $\beta$-actin (dilution, 1:8,000; cat. no. 60008-1; ProteinTech Group, Inc.). Following primary incubation, the membranes were incubated with goat anti-rabbit (dilution, 1:6,000, cat. no. SA00001-2; 
Table I. Sequences of siRNA-LGR6, miR-1236-3p mimics and negative controls.

\begin{tabular}{|c|c|}
\hline RNA & Sequence (5' to $3^{\prime}$ ) \\
\hline \multirow[t]{2}{*}{ siRNA-LGR6 } & Sense: CCUGGAACUGUCUCACAAUTT \\
\hline & Antisense: AUUGUGAGACAGUUCCAGGTT \\
\hline \multirow[t]{2}{*}{ siRNA-Ctrl } & Sense: UUCUCCGAACGUGUCACGUTT \\
\hline & Antisense: ACGUGACACGUUCGGAGAATT \\
\hline $\begin{array}{l}\text { miR-1236-3p } \\
\text { mimics }\end{array}$ & CCUCUUCCCCUUGUCUCUCCAG \\
\hline miR-Ctrl & UUCUCCGAACGUGUCACGUTT \\
\hline
\end{tabular}

LGR6, leucine-rich repeat-containing G-protein coupled receptor 6; siRNA-LGR6, small interfering RNA targeting LGR6; miR, microRNA; Ctrl, control.

ProteinTech Group, Inc.) or donkey anti-mouse (dilution, 1:8,000, cat. no. 715-005-150; Jackson ImmunoResearch) IgG horseradish peroxidase-conjugated secondary antibodies for $1 \mathrm{~h}$ at room temperature. Immunoreactive bands were visualized using a chemiluminescence kit (Thermo Fisher Scientific, Inc.). Protein expression was quantified using ImageJ software (National Institutes of Health) with $\beta$-actin as the loading control.

RNA isolation and reverse transcription-quantitative PCR. Total RNA was extracted from transfected cells using TRIzol ${ }^{\circledR}$ (Thermo Fisher Scientific, Inc.) according to the manufacturer's protocol. Total RNA was reverse transcribed into cDNA using the Hifair ${ }^{\circledR}$ II 1st Strand cDNA Synthesis kit (Shanghai Yeasen Biotechnology Co., Ltd.) or Hairpin-it ${ }^{\mathrm{TM}}$ miRNA RT-PCR Quantitation kit (Shanghai GenePharma Co., Ltd.). Subsequently, qPCR was performed using SYBR Green Select Master Mix (Thermo Fisher Scientific, Inc.) and an ABI 7500 system (Applied Biosystems; Thermo Fisher Scientific, Inc.). The thermocycling conditions were as follows: $95^{\circ} \mathrm{C}$ for $5 \mathrm{~min}$ followed by 40 cycles at $95^{\circ} \mathrm{C}$ for $10 \mathrm{sec}, 58^{\circ} \mathrm{C}$ for $20 \mathrm{sec}$, $72^{\circ} \mathrm{C}$ for $20 \mathrm{sec}$, followed by melting curve detection at $95^{\circ} \mathrm{C}$ for $15 \mathrm{sec}, 60^{\circ} \mathrm{C}$ for $1 \mathrm{~min}$ and $95^{\circ} \mathrm{C}$ for $15 \mathrm{sec}$. The sequences of the primers used for qPCR are listed in Table II. miRNA and mRNA expression levels were quantified using the $2^{-\Delta \Delta C q}$ method (29) and normalized to the internal reference genes U6 and $\beta$-actin.

Statistical analysis. Data are presented as the mean \pm SD. Experiments were performed in triplicate. One-way ANOVA followed by Tukey's post hoc test was used to analyze comparisons among multiple groups. Comparisons between two groups were analyzed using the Student's t-test. $\mathrm{P}<0.05$ was considered to indicate a statistically significant difference.

\section{Results}

LGR6 promotes GBM cell viability and invasion. The expression of LGR6 was detected in GBM cells and normal glial HEB cells. The results indicated that LGR6 mRNA levels were significantly increased in U251 and SHG-44 cells compared
Table II. Sequences of primers used for reverse transcriptionquantitative PCR.

\begin{tabular}{ll}
\hline Gene & \multicolumn{1}{c}{ Sequence (5'-3') } \\
\hline LGR6 & F: ACCCCCTGACGGCTTACCT \\
& R: GCTTGTCCTGGGATGTGTGAG \\
miR-1236-3p & F: CCAATCAGCCTCTTCCCCTT \\
& R: TATGGTTGTTCACGACTCCTTCAC \\
U6 & F: ATTGGAACGATACAGAGAAGATT \\
\multirow{3}{*}{-actin } & R: GGAACGCTTCACGAATTTG \\
& F: CTTAGTTGCGTTACACCCTTTCTTG \\
& R: CTGTCACCTTCACCGTTCCAGTTT
\end{tabular}

LGR6, leucine-rich repeat-containing G-protein coupled receptor 6; miR, microRNA.

with HEB cells (Fig. 1A) and that LGR6 protein expression was increased in SHG-44, U251 and T98G cells compared with HEB cells (Fig. 1B). Additionally, SHG-44 and U251 cells exhibited higher LGR6 mRNA and protein expression levels compared with U87 and T98G cells (Fig. 1A, C and D); therefore, SHG-44 and U251 cells were selected for further experiments. In addition, siRNA-LGR6 significantly reduced LGR6 mRNA and protein expression levels compared with siRNA-Ctrl (Fig. 1B, E and F).

The effect of LGR6 knockdown on SHG-44 and U251 cell viability was investigated. The results indicated that LGR6 knockdown significantly reduced SHG-44 and U251 cell viability at $48 \mathrm{~h}$ compared with the siRNA-Ctrl group (Fig. 2A and B). Additionally, due to the invasive capability of glioma cells that induce malignancy or intracranial metastasis (30), the effect of LGR6 on cell invasion was assessed. The results suggested that LGR6 knockdown significantly reduced the number of invasive cells compared with the siRNA-Ctrl group (Fig. 2C-E).

Conversely, LGR6 overexpression significantly increased the expression levels of LGR6 in SHG-44 and U251 cells compared with the vector group (Fig. 3A-C). Furthermore, LGR6 overexpression significantly increased cell viability compared with the vector group (Fig. $3 \mathrm{H}$ and I) and promoted cell cycle progression. By contrast, LGR6 knockdown arrested the cell cycle at the S phase (Fig. 3D-G). The results suggested that LGR6 served a vital role in regulating GBM cell viability and invasion.

LGR6 mediates TMZ sensitivity in GBM cells. A TMZ-resistant GBM cell model was successfully established and used to investigate TMZ sensitization. A total of 2 TMZ-resistant human glioma cell sublines, SHG-44TMZ+ and U251TMZ+, were generated by increasing TMZ concentrations for 6 months. The $\mathrm{IC}_{50}$ of SHG-44TMZ+ and U251TMZ+ exhibited a $>2$-fold increase compared with parental TMZ-sensitive cell lines (SHG-44TMZ- and U251TMZ- cells; Fig. 4A and B). Moreover, TMZ-resistant SHG-44 and U251 GBM cells displayed increased expression levels of LGR6 compared with TMZ-sensitive SHG-44 and U251 GBM cells (Fig. 4C and D). TMZ-resistant GBM cells displayed higher 
A

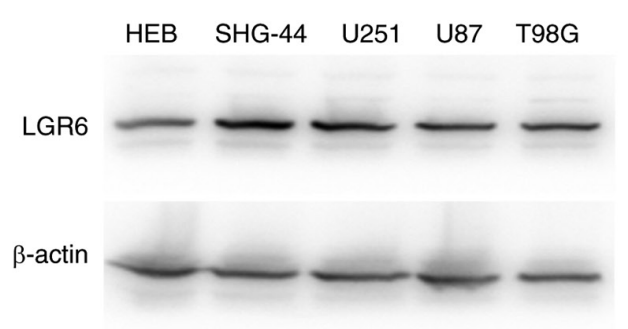

B

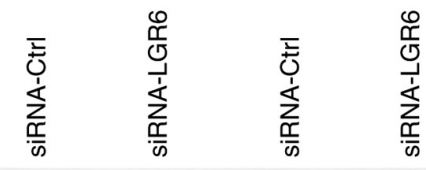

LGR6

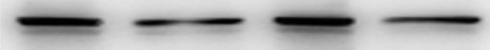

$\beta$-actin

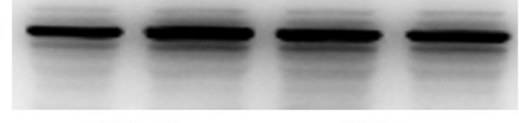

SHG-44

U251
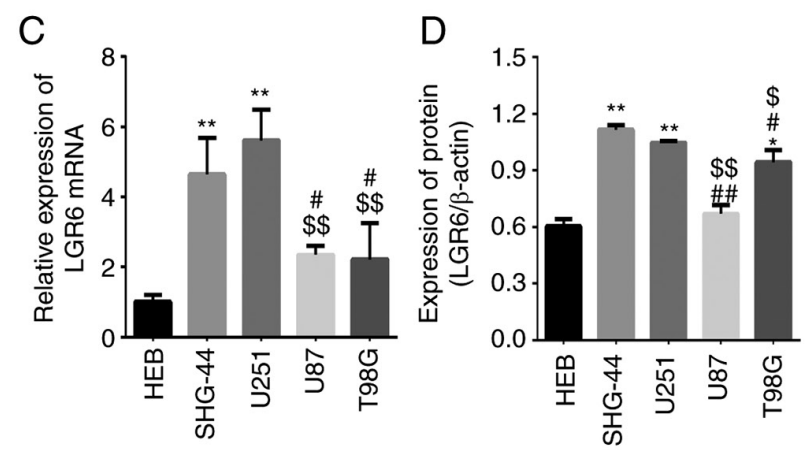

E
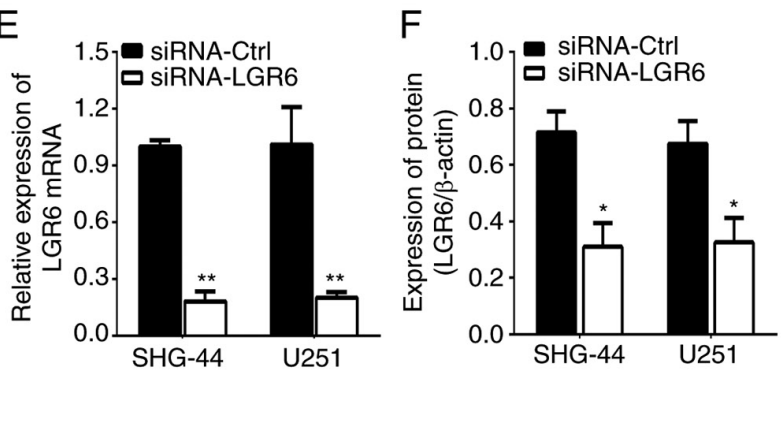

Figure 1. LGR6 expression in GBM cells. Protein expression levels of LGR6 in (A) GBM cells and (B) LGR6-knockdown SHG-44 and U251 cells were determined by western blotting. LGR6 (C) mRNA and (D) protein expression levels in GBM cells. LGR6 (E) mRNA and (F) protein expression levels in LGR6-knockdown SHG-44 and U251 cells. ${ }^{*} \mathrm{P}<0.05,{ }^{* *} \mathrm{P}<0.01$ vs. HEB cells or siRNA-Ctrl; ${ }^{\# \mathrm{P}}<0.05,{ }^{\# \#} \mathrm{P}<0.01$ vs. SHG-44 cells; ${ }^{\$} \mathrm{P}<0.05,{ }^{\$ \$} \mathrm{P}<0.01$ vs. U251 cells; LGR6, leucine-rich repeat-containing G-protein coupled receptor 6; GBM, glioblastoma multiforme; siRNA, small interfering RNA; Ctrl, control.

A

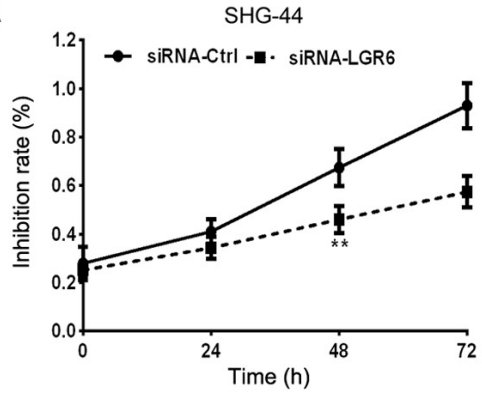

B

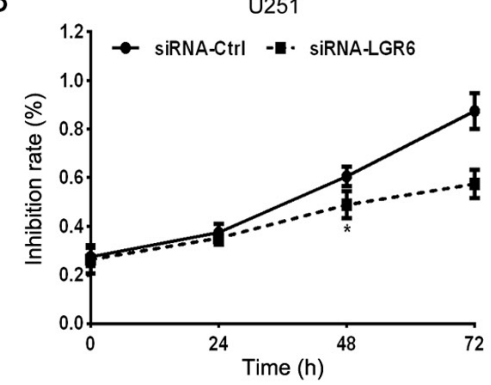

C

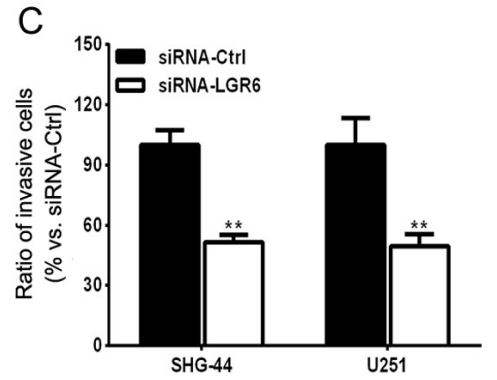

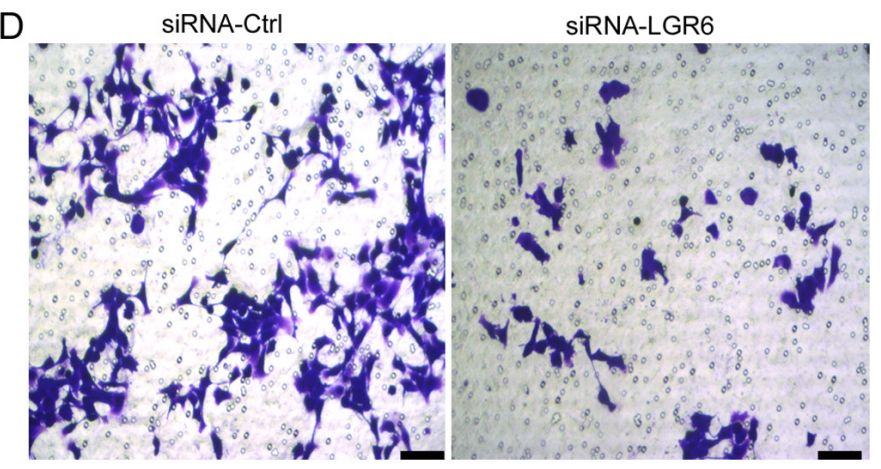

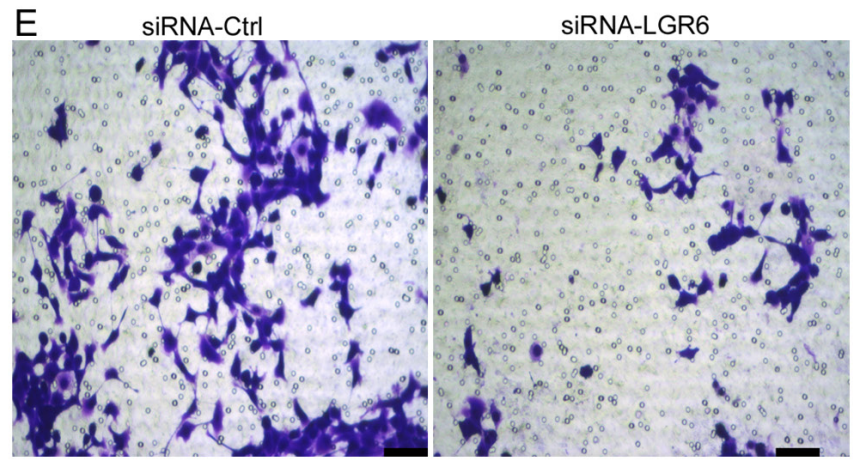

Figure 2. LGR6 knockdown inhibits glioblastoma multiforme cell viability and invasion. Effect of LGR6 knockdown on (A) SHG-44 and (B) U251 cell viability. (C) Effect of LGR6 knockdown on cell invasion in (D) SHG-44 and (E) U251 cells. Scale bar, $200 \mu \mathrm{m}$. Magnification, $\mathrm{x} 200 .{ }^{*} \mathrm{P}<0.05,{ }^{* *} \mathrm{P}<0.01$ vs. siRNA-Ctrl. LGR6, leucine-rich repeat-containing G-protein coupled receptor 6; siRNA, small interfering RNA; Ctrl, control.

viability rates compared with TMZ-sensitive cells following treatment with a series of TMZ concentrations $(0,100,200$, 300,400 and $500 \mu \mathrm{M}$; Fig. 4C and D). U251 and SHG-44 cell viability decreased in a time-dependent manner, whereas LGR6 knockdown decreased U251 and SHG-44 cell viability compared with the siRNA-Ctrl group (Fig. 4E-H). Based on 
A

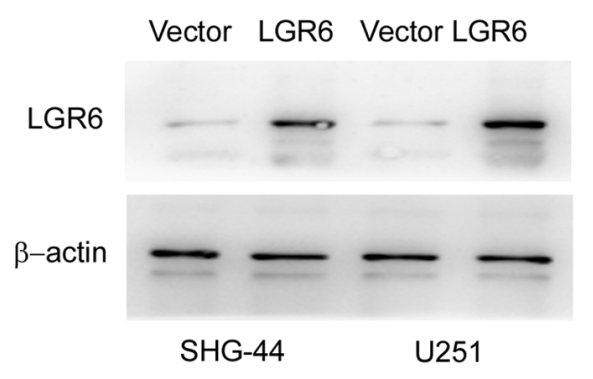

D SHG-44

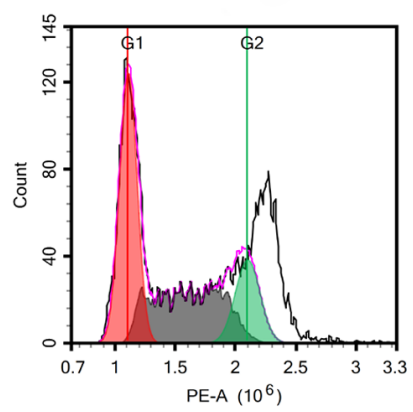

E

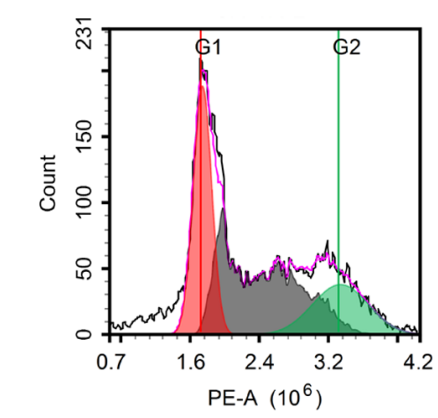

F

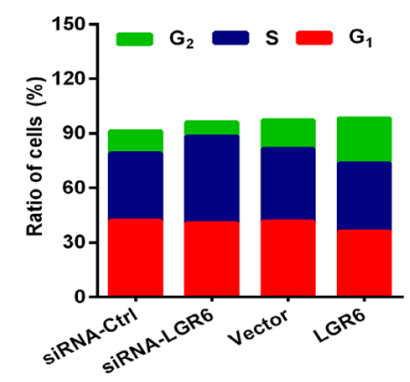

B

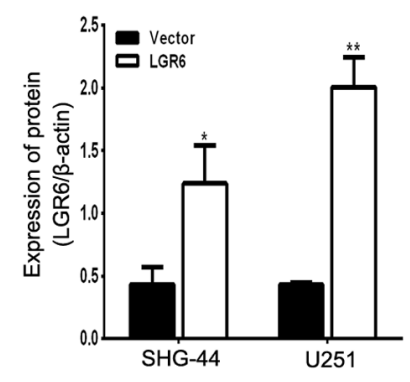

C
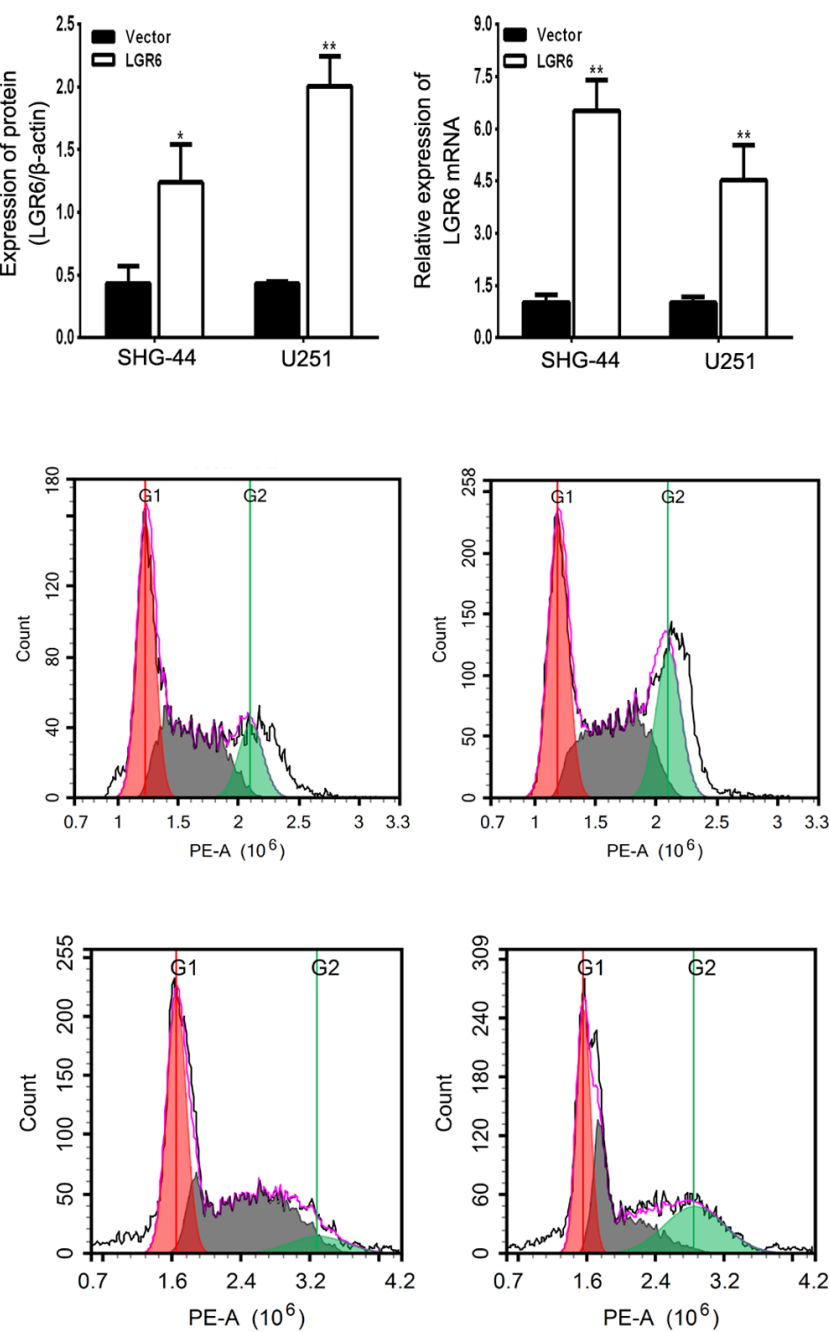

H SHG-44

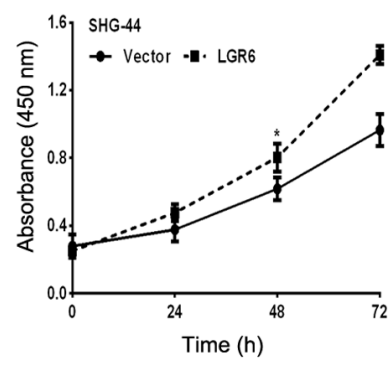

I U251
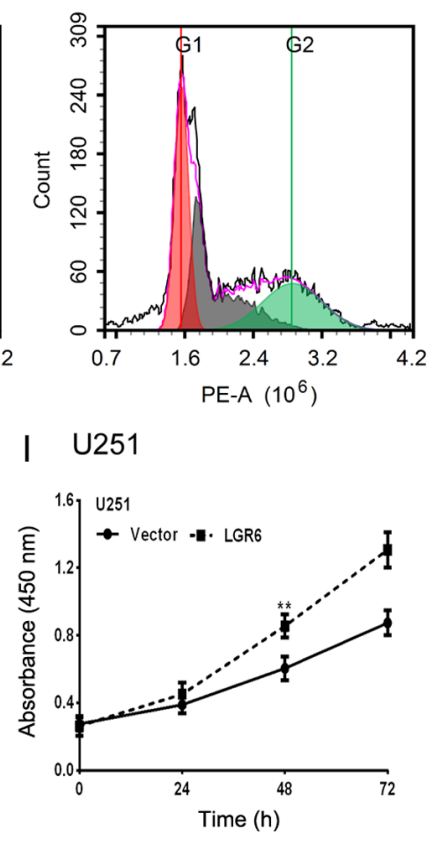

Figure 3. LGR6 promotes cell viability and cell cycle progression. LGR6 protein expression levels were (A) determined by western blotting and (B) semi-quantified following LGR6 overexpression. (C) LGR6 mRNA expression levels following LGR6 overexpression. *P<0.05, ${ }^{* *} \mathrm{P}<0.01$ vs. vector. Cell cycle distribution was determined via flow cytometry in (D) SHG-44 and (E) U251 cells, and quantified for (F) SHG-44 and (G) U251 cells. Cell viability in (H) SHG-44 and (I) U251 cells following LGR6 overexpression. ${ }^{*} \mathrm{P}<0.05,{ }^{* *} \mathrm{P}<0.01$ vs. vector. LGR6, leucine-rich repeat-containing G-protein coupled receptor 6; siRNA, small interfering RNA; Ctrl, control.

the results, it was hypothesized that LGR6 participated in the failure of TMZ chemotherapy in GBM, which might indicate a new therapeutic target for the disease.

LGR6 promotes GBM viability and chemoresistance by activating Akt signaling. The Akt signaling pathway is involved in numerous types of cancer, including $\operatorname{GBM}(31,32)$; therefore, the levels of p- and total Akt in transfected GBM cells were measured. The results indicated that LGR6 overexpression significantly increased the levels of p-Akt compared with the vector group, but did not alter the total levels of Akt (Fig. 5A and B), which suggested that LGR6 might activate Akt signaling to mediate GBM malignancy. Therefore, it was hypothesized that as LGR6 induced the activation of Akt signaling during GBM progression, the loss of Akt activity may abolish the regulatory ability of LGR6.

Further experiments were conducted to investigate whether MK-2206, a specific inhibitor of Akt signaling, reversed 
A

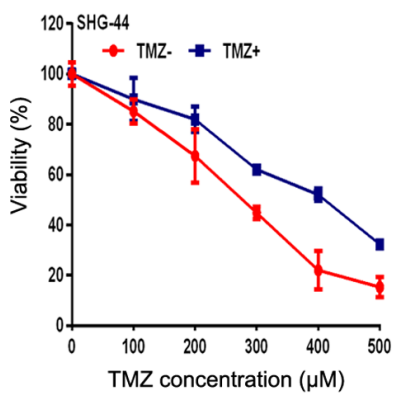

E

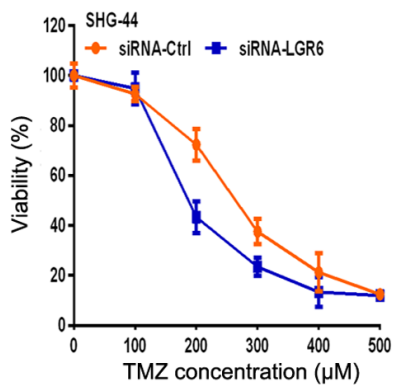

B

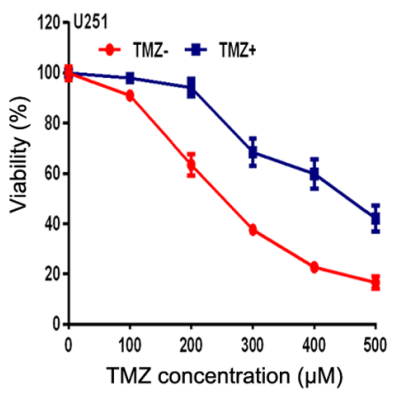

$\mathrm{F}$

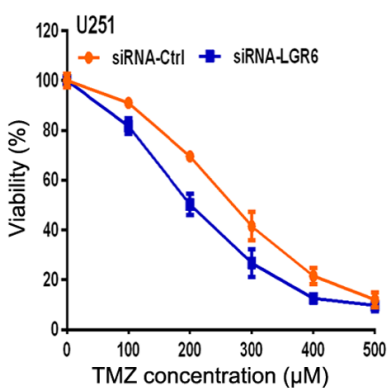

C

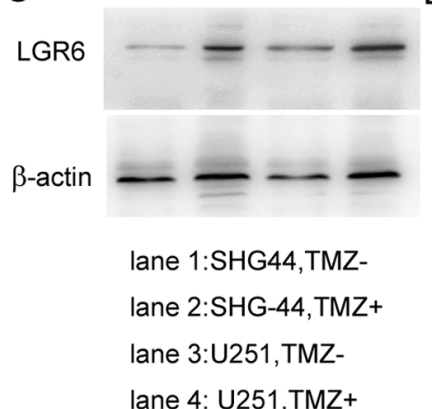

G

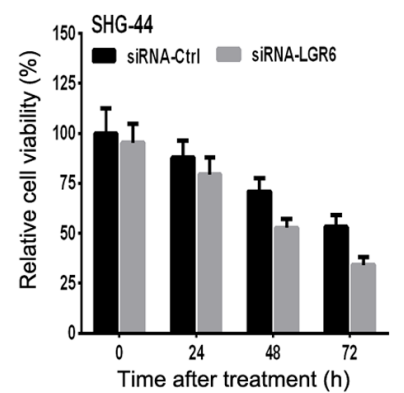

D

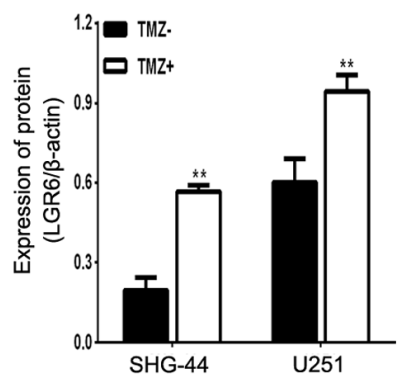

$\mathrm{H}$

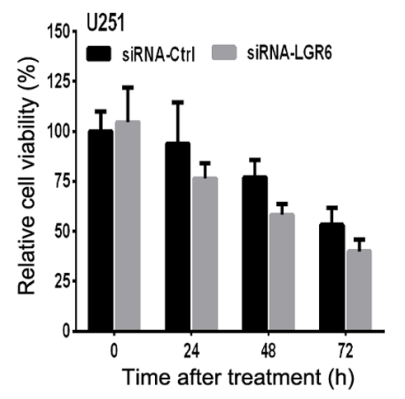

Figure 4. LGR6 enhances glioblastoma multiforme cell chemoresistance. Cell viability in TMZ-resistant (A) SHG-44 and (B) U251 cells. LGR6 protein expression was (C) determined by western blotting and (D) semi-quantified in TMZ-resistant and -sensitive cells. Cell viability in LGR6-knockdown TMZ-resistant (E) SHG-44 and (F) U251 cells. Cell viability in LGR6-knockdown (G) SHG-44 and (H) U251 cells treated with $200 \mu$ M TMZ at different time points. ${ }^{* *} \mathrm{P}<0.01$ vs. TMZ-sensitive cells. LGR6, leucine-rich repeat-containing G-protein coupled receptor 6; TMZ, temozolomide; siRNA, small interfering RNA; Ctrl, control.

LGR6-induced cell viability and reduced cell viability in response to TMZ treatment in SHG-44 and U251 cells. The results were consistent with the hypothesis (Fig. 5C-F), which suggested that LGR6 promoted GBM viability and chemoresistance by activating Akt signaling.

LGR6 is a target of miR-1236-3p. miR-1236-3p serves as a tumor suppressor in various types of cancer (33-35). To investigate whether LGR6 was a potential target of miR-1236-3p, the available complementary-based algorisms were predicted using TargetScan and miRTarBase. The results indicated that miR-1236-3p expression levels were significantly decreased in U251 and SHG-44 cells compared with HEB cells and SHG-44 and U251 cells displayed lower miR-1236-3p levels compared with U87 and T98G cells (Fig. 6A). Additionally, miR-1236-3p mimic significantly increased the expression of miR-1236-3p and significantly decreased LGR6 expression levels at the mRNA and protein level compared with miR-Ctrl (Fig. 6B-D). Based on the predicted targeting sites of miR-1236-3p, LGR6 3'-UTR WT and Mut luciferase reporter plasmids were constructed. The results indicated that miR-1236-3p mimic significantly decreased the luciferase activity of LGR6 WT 3'-UTR compared with miR-Ctrl, but did not alter the luciferase activity of LGR6 Mut 3'-UTR (Fig. 6E and F). The results suggested that LGR6 was an miR-1236-3p target, which may mediate its effects during cancer development.

\section{Discussion}

As the most prevalent and malignant brain tumor in the adult central nervous system (36), glioma results in a high number of brain tumor-related deaths each year (37). Since the present curative efficiency on glioma is limited, developing novel therapeutic targets and understanding the molecular mechanism underlying glioma progression is important. Accumulating evidence has demonstrated that LGR6 is a contributing factor to cell proliferation in multiple types of human cancer, including gastric cancer and colon cancer $(8,10)$; however, its role in glioma is not completely understood. In the present study, although the expression of LGR6 in glioma tissues was not investigated, in vitro experiments indicated that LGR6 expression was higher in GBM cell lines compared with the normal glial cell line and SHG-44 and U251 cells displayed higher LGR6 expression levels compared with U87 and T98G cells. In addition, LGR6 knockdown inhibited SHG-44 and U251 cell viability compared with the siRNA-Ctrl group. Additionally, TMZ-resistant SHG-44 and U251 cells displayed increased LGR6 expression levels compared with TMZ-sensitive cells. To the best of our knowledge, the present study was the first to suggest that LGR6 may be associated with cell viability and TMZ resistance in GBM.

LGR4, LGR5 and LGR6 are receptors of the R-spondin protein family (38-40). In vitro experiments have demonstrated that the three proteins could bind all types of R-spondins (40). Lebensohn and Rohatgi (41) indicated that R-spondin 1 binding to LGR4/5/6 is essential for WNT signaling. Chong et al (42) proposed that WNT can activate Akt directly or via WNT1-induced secreted protein. Both Akt and $\mathrm{WNT} / \beta$-catenin signaling pathways may regulate cell proliferation and migration (42-45), and serve important roles in GBM (46). In accordance with the finding that the 
A

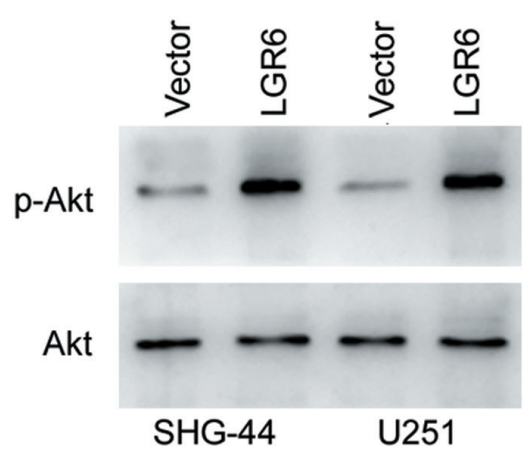

C

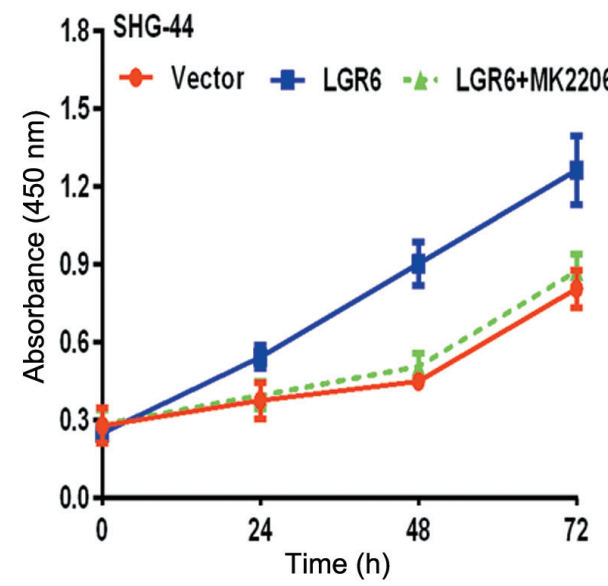

$E$

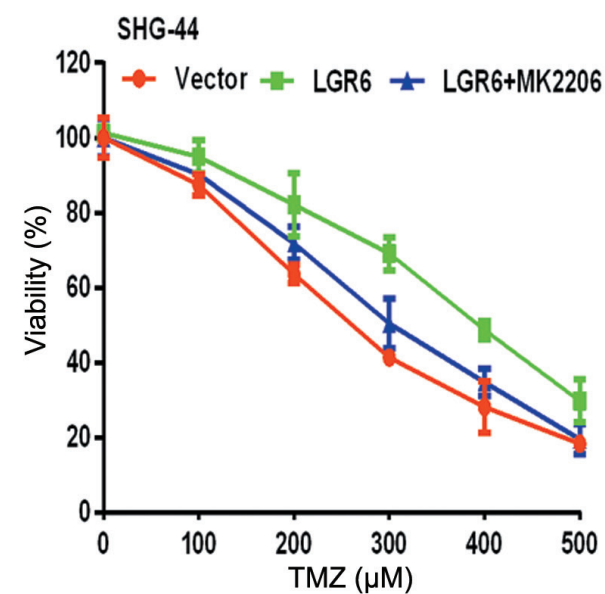

B

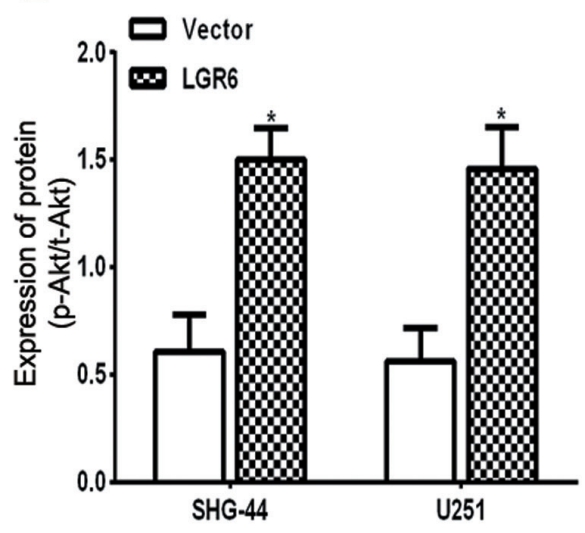

D

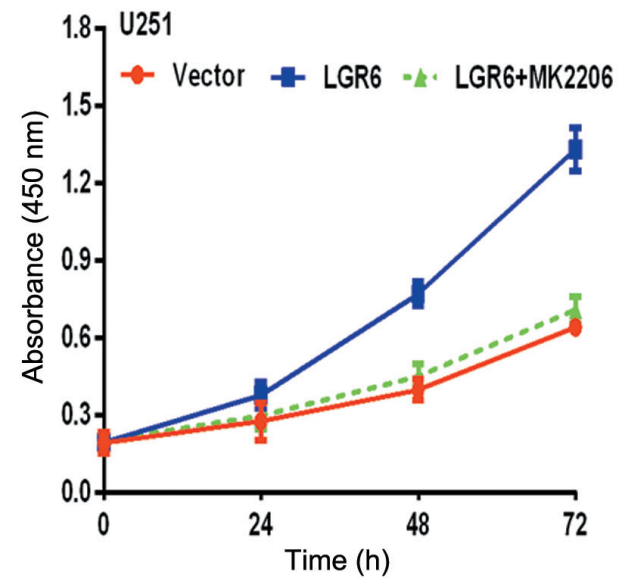

$\mathrm{F}$

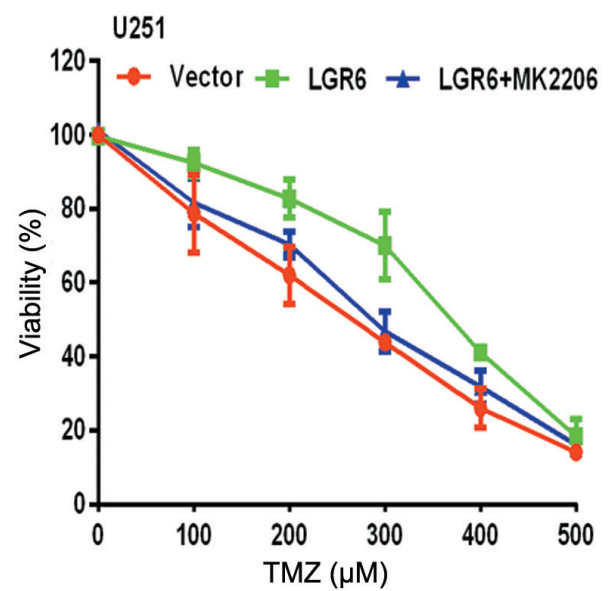

Figure 5. LGR6 regulates glioblastoma multiforme cell chemoresistance via the Akt signaling pathway. Following LGR6 overexpression, protein expression levels were (A) determined by western blotting and (B) the ratio of p-Akt/Akt was semi-quantified. Cell viability of LGR6-overexpression (C) SHG-44 and (D) U251 cells in the absence or presence of MK2206. Cell viability of LGR6-overexpression (E) SHG-44 and (F) U251 cells in the absence or presence of MK2206 and different concentrations of TMZ. "P<0.05 vs. vector. LGR6, leucine-rich repeat-containing G-protein coupled receptor 6; p, phosphorylated; TMZ, temozolomide.

Akt signaling pathway is activated in the TMZ-resistant U87 cell line (46), the present study indicated that overexpression of LGR6 also increased the levels of phosphorylated Akt in TMZ-resistant cell lines. The results of the present study combined with the results of previous reports indicated that
LGR6 may serve an important role in TMZ-resistant GBM, which may be mediated via the Akt signaling pathway.

Previous studies have reported that miRs serve important roles in the majority of different types of cancer by modulating key processes during tumorigenesis $(47,48)$. Through controlling 
A

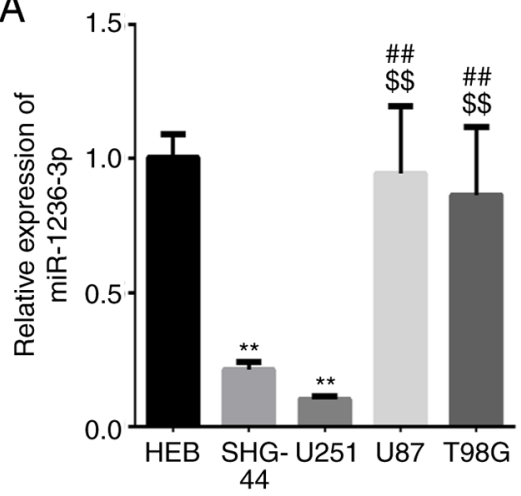

B

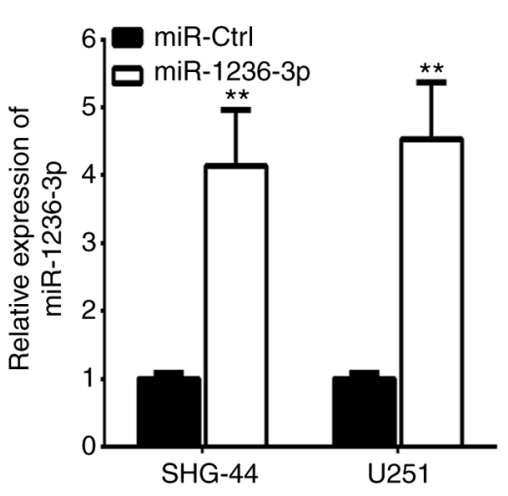

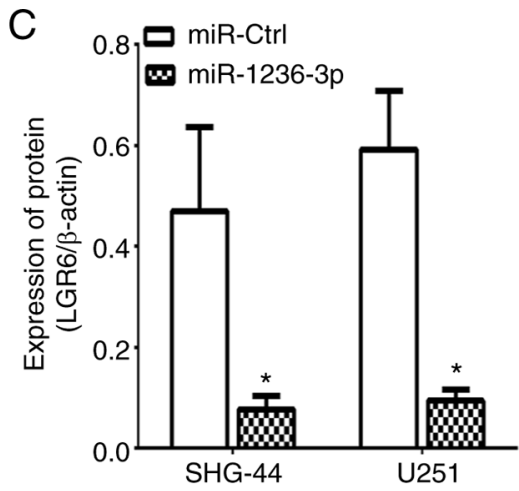

$\mathrm{F}$

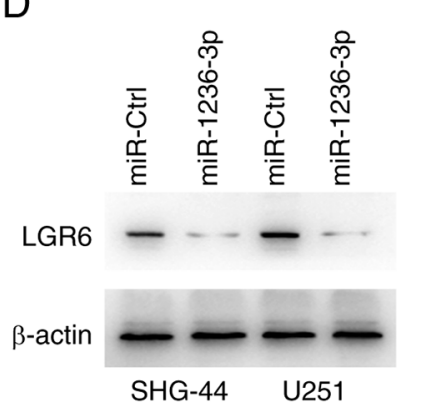

E

LGR6·3'-UTR·wild.type …...................... TAAGCTTTGGAAGAGATTACACA-3' $4 \quad|||| \mid$ miR-1236-3p..3'-CCUCUUCCCCUUGUCUCUCCAG-5' ...5'...TAAGCTTTCCUUGAGATTACACA-3' لـ

LGR6.3'-UTR.mutant

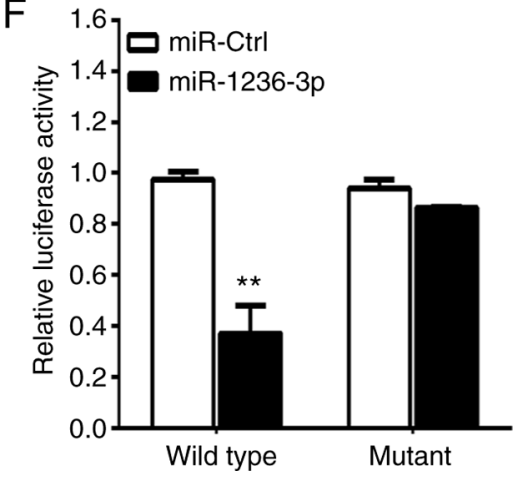

Figure 6. LGR6 is targeted by miR-1236-3p. miR-1236-3p expression levels in (A) glioblastoma multiforme cells and (B) miR-1236-3p mimic-transfected cells. ${ }^{* *} \mathrm{P}<0.01$ vs. miR-Ctrl or HEB cells; ${ }^{\# \#} \mathrm{P}<0.01$ vs. SHG-44 cells; ${ }^{\$ \$} \mathrm{P}<0.01$ vs. U251 cells; Following miR-1236-3p mimic transfection, (C) LGR6 protein expression levels were (D) determined by western blotting. (E) Potential binding site between LGR6 and miR-1236-3p. (F) Luciferase activity of wild-type or mutant LGR6 3'-UTR luciferase reporter constructs. ${ }^{*} \mathrm{P}<0.05,{ }^{* *} \mathrm{P}<0.01$ vs. miR-Ctrl. LGR6, leucine-rich repeat-containing G-protein coupled receptor 6; miR, microRNA; Ctrl, control; 3'UTR, 3'-untranslated region.

the gene expression of target mRNAs, miRs can serve as oncogenes or tumor suppressor genes (49). miR-1236-3p, an intronic miRNA, is involved in multiple types of cancer, such as gastric (50,51), ovarian (52), lung (34) and bladder (53) cancer. Wang et al (54) indicated that miR-1236-3p is prominently downregulated in DDP-resistant A549 cells, the role of which in lung cancer cells may be mediated by modulation of tumor protein, translationally-controlled 1 and inhibition of the Pim-3 proto-oncogene, serine/threonine kinase signaling pathway. In the present study, LGR6 was predicted as the potential target of miR-1236-3p by bioinformatics analysis. The luciferase reporter assays indicated that miR-1236-3p regulated LGR6 expression levels by targeting its 3'-UTR sequence and miR-1236-3p was downregulated in GBM cells compared with HEB cells. Moreover, miR-1236-3p overexpression decreased LGR6 expression levels compared with control cells, which suggested that LGR6 might be a downstream effect effector of miR-1236-3p. Similarly, a previous study indicated miR-1236-3p suppressed the progression of glioma by targeting homeobox B7 (HOXB7), a key factor for tumor-associated angiogenic switch $(55,56)$. Previous studies have indicated that HOXB7 is involved in cancer stem cell biology by regulating the expression of the stem cell-related gene, such as lin-28 homolog B (57) and run-related transcription factor 2 (RUNX2) (58). By contrast, LGR6 ${ }^{+}$cancer cells display self-renewal and differentiation capacities, alongside higher oncogenic potential in lung cancer (59). Therefore, whether the HOXB7/LGR6 axis is involved in regulating glioma stem cells requires further investigation.
In conclusion, to the best of our knowledge, the present study identified the essential roles of LGR6 in glioma for the first time. In addition, the results indicated a functional mechanism underlying LGR6 and suggested that the miR-1236-3p/LGR6/Akt signaling axis regulated the sensitivity of GBM cells to TMZ. The results of the present study indicated a potential mechanism underlying the recurrence and resistance to glioma therapies and suggested a potential cellular and molecular therapeutic target for GBM.

\section{Acknowledgements}

Not applicable.

\section{Funding}

No funding was received.

\section{Availability of data and materials}

The datasets used and/or analyzed during the present study are available from the corresponding author on reasonable request.

\section{Authors' contributions}

YC, XY and FX designed the present study. YC, XY, XG, SS and MY collected, analyzed and interpreted data. YC, XY, 
XG, FX and MY drafted and reviewed the manuscript. MY, FX and SS revised the manuscript and provided material support. All authors agreed to be accountable for all aspects of the current work. All authors read and approved the final manuscript.

\section{Ethics approval and consent to participate}

Not applicable.

\section{Patient consent for publication}

Not applicable.

\section{Competing interests}

The authors declare that they have no competing interests.

\section{References}

1. Jia B, Liu W, Gu J, Wang J, Lv W, Zhang W, Hao Q, Pang Z, Mu N, Zhang W and Guo Q: MiR-7-5p suppresses stemness and enhances temozolomide sensitivity of drug-resistant glioblastoma cells by targeting Yin Yang 1. Exp Cell Res 375: 73-81, 2019.

2. McLendon R, Friedman AH, Bigner D, Van Meir EG, Brat DJ, Mastrogianakis G, Olson JJ, Mikkelsen T, Lehman N, Aldape $\mathrm{K}$, et al: Comprehensive genomic characterization defines human glioblastoma genes and core pathways. Nature 455: 1061-1068, 2008.

3. Shi F, Guo H, Zhang R, Liu H, Wu L, Wu Q, Liu J, Liu T and Zhang Q: The PI3K inhibitor GDC-0941 enhances radiosensitization and reduces chemoresistance to temozolomide in GBM cell lines. Neuroscience 346: 298-308, 2017.

4. Schreck KC and Grossman SA: Role of temozolomide in the treatment of cancers involving the central nervous system. Oncology (Williston Park) 32: 555-560, 569, 2018.

5. Stephen ZR, Kievit FM, Veiseh O, Chiarelli PA, Fang C, Wang K, Hatzinger SJ, Ellenbogen RG, Silber JR and Zhang M: Redox-responsive magnetic nanoparticle for targeted convection-enhanced delivery of O6-benzylguanine to brain tumors. ACS Nano 8: 10383-10395, 2014.

6. Stavrovskaya AA, Shushanov SS and Rybalkina EY: Problems of glioblastoma multiforme drug resistance. Biochemistry (Mosc) 81: 91-100, 2016.

7. Choi S, Yu Y, Grimmer MR, Wahl M, Chang SM and Costello JF: Temozolomide-associated hypermutation in gliomas. Neuro Oncol 20: 1300-1309, 2018.

8. Wang F, Dai CQ, Zhang LR, Bing C, Qin J and Liu YF: Downregulation of Lgr6 inhibits proliferation and invasion and increases apoptosis in human colorectal cancer. Int J Mol Med 42: 625-632, 2018.

9. Wang W, Ding S, Zhang H, Li J, Zhan J and Zhang H: G protein-coupled receptor LGR6 is an independent risk factor for colon adenocarcinoma. Front Med 13: 482-491, 2019.

10. Ke J, Ma P, Chen J, Qin J and Qian H: LGR6 promotes the progression of gastric cancer through PI3K/AKT/mTOR pathway. Onco Targets Ther 11: 3025-3033, 2018.

11. Kazanskaya O, Glinka A, del Barco Barrantes I, Stannek P Niehrs C and Wu W: R-Spondin2 is a secreted activator of $\mathrm{Wnt} / \mathrm{beta}$-catenin signaling and is required for xenopus myogenesis. Dev Cell 7: 525-534, 2004.

12. Kim KA, Zhao J, Andarmani S, Kakitani M, Oshima T, Binnerts ME, Abo A, Tomizuka K and Funk WD: R-Spondin proteins: A novel link to beta-catenin activation. Cell Cycle 5: 23-26, 2006.

13. Nam JS, Turcotte TJ, Smith PF, Choi S and Yoon JK: Mouse cristin/R-spondin family proteins are novel ligands for the Frizzled 8 and LRP6 receptors and activate beta-catenindependent gene expression. J Biol Chem 281: 13247-13257, 2006.

14. Raslan AA and Yoon JK: R-spondins: Multi-mode WNT signaling regulators in adult stem cells. Int J Biochem Cell Biol 106: 26-34, 2019.
15. Zhang Y, Guo L, Lu X, Cheng C, Sun S, Li W, Zhao L, Lai C, Zhang S, Yu C, et al: Characterization of Lgr6+ cells as an enriched population of hair cell progenitors compared to Lgr5+ cells for hair cell generation in the neonatal mouse cochlea. Front Mol Neurosci 11: 147, 2018.

16. Szenker-Ravi E, Altunoglu U, Leushacke M, Bosso-Lefèvre C, Khatoo M, Thi Tran H, Naert T, Noelanders R, Hajamohideen A, Beneteau C, et al: RSPO2 inhibition of RNF43 and ZNRF3 governs limb development independently of LGR4/5/6. Nature 557: 564-569, 2018.

17. Schindler AJ, Watanabe A and Howell SB: LGR5 and LGR6 in stem cell biology and ovarian cancer. Oncotarget 9: 1346-1355, 2017.

18. Bin BH, Bhin J, Takaishi M, Toyoshima KE, Kawamata S, Ito K, Hara T, Watanabe T, Irié T, Takagishi T, et al: Requirement of zinc transporter ZIP10 for epidermal development: Implication of the ZIP10-p63 axis in epithelial homeostasis. Proc Natl Acad Sci USA 114: 12243-12248, 2017

19. Guinot A, Oeztuerk-Winder F and Ventura JJ: miR-17-92/p38 $\alpha$ dysregulation enhances Wnt signaling and selects Lgr6+ cancer stem-like cells during lung adenocarcinoma progression. Cancer Res 76: 4012-4022, 2016.

20. Roos A, Dhruv HD, Peng S, Inge LJ, Tuncali S, Pineda M, Millard N, Mayo Z, Eschbacher JM, Loftus JC, et al: EGFRvIII-stat5 signaling enhances glioblastoma cell migration and survival. Mol Cancer Res 16: 1185-1195, 2018.

21. Huang W, Ding X, Ye H, Wang J, Shao J and Huang T: Hypoxia enhances the migration and invasion of human glioblastoma U87 cells through PI3K/Akt/mTOR/HIF-1 $\alpha$ pathway. Neuroreport 29: 1578-1585, 2018.

22. Wang L, Wang J, Jin T, Zhou Y and Chen Q: FoxG1 facilitates proliferation and inhibits differentiation by downregulating FoxO/Smad signaling in glioblastoma. Biochem Biophys Res Commun 504: 46-53, 2018.

23. Vainchenker W, Leroy E, Gilles L, Marty C, Plo I and Constantinescu SN: JAK inhibitors for the treatment of myeloproliferative neoplasms and other disorders. F1000Res 7: 82, 2018.

24. Berendsen S, Spliet WGM, Geurts M, Van Hecke W, Seute T, Snijders TJ, Bours V, Bell EH, Chakravarti A and Robe PA: Epilepsy associates with decreased HIF-1 $\alpha /$ STAT5b signaling in glioblastoma. Cancers (Basel) 11: 41, 2019.

25. Shen X, Zhang J, Zhang X, Wang Y, Hu Y and Guo J: Retinoic acid-induced protein 14 (RAI14) promotes mTOR-mediated inflammation under inflammatory stress and chemical hypoxia in a U87 glioblastoma cell line. Cell Mol Neurobiol 39: 241-254, 2019.

26. Ke XX, Pang Y, Chen K, Zhang D, Wang F, Zhu S, Mao J, Hu X, Zhang $\mathrm{G}$ and Cui H: Knockdown of arsenic resistance protein 2 inhibits human glioblastoma cell proliferation through the MAPK/ERK pathway. Oncol Rep 40: 3313-3322, 2018.

27. Cheng P, Ma Y, Gao Z and Duan L: High mobility group box 1 (HMGB1) predicts invasion and poor prognosis of glioblastoma multiforme via activating AKT signaling in an autocrine pathway. Med Sci Monit 24: 8916-8924, 2018.

28. Nadkarni A, Shrivastav M, Mladek AC, Schwingler PM, Grogan PT, Chen J and Sarkaria JN: ATM inhibitor KU-55933 increases the TMZ responsiveness of only inherently TMZ sensitive GBM cells. J Neurooncol 110: 349-357, 2012.

29. Livak KJ and Schmittgen TD: Analysis of relative gene expression data using real-time quantitative PCR and the 2(-Delta Delta C(T)) method. Methods 25: 402-408, 2001.

30. Sun Z, Xue H, Wei Y, Wang C, Yu R, Wang C, Wang S, Xu J, Qian M, Meng Q and Li G: Mucin O-glycosylating enzyme GALNT2 facilitates the malignant character of glioma by activating the EGFR/PI3K/Akt/mTOR axis. Clin Sci (Lond) 133: 1167-1184, 2019.

31. Wen YT, Wu AT, Bamodu OA, Wei L, Lin CM, Yen Y, Chao TY, Mukhopadhyay D, Hsiao M and Huang HS: A novel multi-target small molecule, LCC-09, inhibits stemness and therapy-resistant phenotypes of glioblastoma cells by increasing miR-34a and deregulating the DRD4/Akt/mTOR signaling axis. Cancers (Basel) 11: 1442, 2019.

32. Kim EH, Jo Y, Sai S, Park MJ, Kim JY, Kim JS, Lee YJ, Cho JM, Kwak SY, Baek JH, et al: Tumor-treating fields induce autophagy by blocking the Akt2/miR29b axis in glioblastoma cells. Oncogene 38: 6630-6646, 2019.

33. An JX, Ma MH, Zhang CD, Shao S, Zhou NM and Dai DQ: miR-1236-3p inhibits invasion and metastasis in gastric cancer by targeting MTA2. Cancer Cell Int 18: 66, 2018. 
34. Li C, Ge Q, Liu J, Zhang Q, Wang C, Cui K and Chen Z: Effects of miR-1236-3p and miR-370-5p on activation of $\mathrm{p} 21$ in various tumors and its inhibition on the growth of lung cancer cells. Tumour Biol 39: 1010428317710824, 2017.

35. Bian T, Jiang D, Liu J, Yuan X, Feng J, Li Q, Zhang Q, Li X, Liu $\mathrm{Y}$ and Zhang J: miR-1236-3p suppresses the migration and invasion by targeting KLF8 in lung adenocarcinoma A549 cells. Biochem Biophys Res Commun 492: 461-467, 2017.

36. Wen PY and Kesari S: Malignant gliomas in adults. N Engl J Med 359: 492-507, 2008.

37. Torre LA, Bray FI, Siegel RL, Ferlay J, Lortettieulent J and Jemal A: Global cancer statistics, 2012. CA Cancer J Clin 65: 87-108, 2015.

38. Huang PY,Kandyba E, Jabouille A, Sjolund J, Kumar A, Halliwill K, McCreery M, DelRosario R, Kang HC, Wong CE, et al: Lgr6 is a stem cell marker in mouse skin squamous cell carcinoma. Nat Genet 49: 1624-1632, 2017.

39. de Lau W, Barker N, Low TY, Koo BK, Li VS, Teunissen H, Kujala P, Haegebarth A, Peters PJ, van de Wetering M, et al: Lgr5 homologues associate with Wnt receptors and mediate R-spondin signalling. Nature 476: 293-297, 2011.

40. Glinka A, Dolde C, Kirsch N, Huang YL, Kazanskaya O, Ingelfinger D, Boutros M, Cruciat CM and Niehrs C: LGR4 and LGR5 are R-spondin receptors mediating Wnt $/ \beta$-catenin and Wnt/PCP signalling. EMBO Rep 12: 1055-1061, 2011.

41. Lebensohn AM and Rohatgi R: R-spondins can potentiate WNT signaling without LGRs. Elife 7: e33126, 2018.

42. Chong ZZ, Li F and Maiese K: Employing new cellular therapeutic targets for Alzheimer's disease: A change for the better? Curr Neurovasc Res 2: 55-72, 2005.

43. Hers I, Vincent EE and Tavaré JM: Akt signalling in health and disease. Cell Signal 23: 1515-1527, 2011.

44. Luo J, Manning BD and Cantley LC: Targeting the PI3K-Akt pathway in human cancer: Rationale and promise. Cancer Cell 4 257-262, 2003.

45. Angers S and Moon RT: Proximal events in Wnt signal transduction. Nat Rev Mol Cell Biol 10: 468-477, 2009.

46. Yi GZ, Liu YW, Xiang W, Wang H, Chen ZY, Xie SD and Qi ST: Akt and $\beta$-catenin contribute to TMZ resistance and EMT of MGMT negative malignant glioma cell line. J Neurol Sci 367: 101-106, 2016.
47. Hayes J, Peruzzi PP and Lawler S: MicroRNAs in cancer: Biomarkers, functions and therapy. Trends Mol Med 20: 460-469, 2014.

48. Acunzo M, Romano G, Wernicke D and Croce CM: MicroRNA and cancer-a brief overview. Adv Biol Regul 57: 1-9, 2015.

49. Gregory RI and Shiekhattar R: MicroRNA biogenesis and cancer. Cancer Res 65: 3509-3512, 2005.

50. An JX, MaZS, Ma MH, Shao S, Cao FL and Dai DQ: MiR-1236-3p serves as a new diagnostic and prognostic biomarker for gastric cancer. Cancer Biomark 25: 127-132, 2019.

51. Zhu XP, Wang XL, Ma J, Fang YF, Zhang HJ, Zhang C and Feng MC: Down-regulation of miR-1236-3p is correlated with clinical progression and unfavorable prognosis in gastric cancer. Eur Rev Med Pharmacol Sci 22: 5914-5919, 2018.

52. Li QH, Liu Y, Chen S, Zong ZH, Du YP, Sheng XJ and Zhao Y: circ-CSPP1 promotes proliferation, invasion and migration of ovarian cancer cells by acting as a miR-1236-3p sponge. Biomed Pharmacother 114: 108832, 2019.

53. Zhang Q, Miao S, Li C, Cui K, Ge Q and Chen Z: S-phase kinase-associated protein 2 impairs the inhibitory effects of miR-1236-3p on bladder tumors. Am J Transl Res 10: 731-743, 2018.

54. Wang Z, Liu L, Guo X, Guo C and Wang W: microRNA-1236-3p regulates DDP resistance in lung cancer cells. Open Med (Wars) 14: 41-51, 2019

55. Carè A, Felicetti F, Meccia E, Bottero L, Parenza M, Stoppacciaro A, Peschle C and Colombo MP: HOXB7: A key factor for tumor-associated angiogenic switch. Cancer Res 61: 6532-6539, 2001

56. Duan X, Liu D, Wang Y and Chen Z: Circular RNA hsa circ_0074362 Promotes Glioma Cell Proliferation, Migration, and invasion by attenuating the inhibition of miR-1236-3p on HOXB7 expression. DNA Cell Biol 37: 917-924, 2018.

57. Monterisi S, Lo Riso P, Russo K, Bertalot G, Vecchi M, Testa G, Di Fiore PP and Bianchi F: HOXB7 overexpression in lung cancer is a hallmark of acquired stem-like phenotype. Oncogene 37: 3575-3588, 2018.

58. Gao RT, Zhan LP, Meng C, Zhang N, Chang SM, Yao R and Li C: Homeobox B7 promotes the osteogenic differentiation potential of mesenchymal stem cells by activating RUNX2 and transcript of BSP. Int J Clin Exp Med 8: 10459-10470, 2015.

59. Cortesi E and Ventura JJ: Lgr6: From stemness to cancer progression. J Lung Health Dis 3: 12-15, 2019. 